\title{
Author Correction: Variants in exons 5 and 6 of ACTB cause syndromic thrombocytopenia
}

\author{
Sharissa L. Latham (1) ${ }^{1}$, Nadja Ehmke ${ }^{2,3}$, Patrick Y.A. Reinke ${ }^{1,4}$, Manuel H. Taft (1) ${ }^{1}$, Dorothee Eicke ${ }^{5}$, \\ Theresia Reindl', Werner Stenzel ${ }^{6}$, Michael J. Lyons ${ }^{7}$, Michael J. Friez ${ }^{7}$, Jennifer A. Lee ${ }^{7}$, Ramona Hecker ${ }^{8}$, \\ Michael C. Frühwald ${ }^{9}$, Kerstin Becker ${ }^{10}$, Teresa M. Neuhann ${ }^{10}$, Denise Horn ${ }^{2}$, Evelin Schrock ${ }^{11}$, Indra Niehaus ${ }^{11}$, \\ Katharina Sarnow ${ }^{11}$, Konrad Grützmann ${ }^{12}$, Luzie Gawehn ${ }^{11}$, Barbara Klink (D) 11, Andreas Rump ${ }^{11}$, \\ Christine Chaponnier ${ }^{13}$, Constanca Figueiredo ${ }^{5}$, Ralf Knöfler ${ }^{14}$, Dietmar J. Manstein ${ }^{1,4} \&$ Nataliya Di Donato (1) ${ }^{11}$
}

Correction to: Nature Communications https://doi.org/10.1038/s41467-018-06713-0; published online 12 Oct 2018

The original version of this Article contained an error in Fig. 4. In panel i, the lower CYA and a-SMA images were inadvertently inverted. This has been corrected in both the PDF and HTML versions of the Article.

Published online: 19 November 2018

\begin{abstract}
reproduction in any medium or format, as long as you give appropriate credit to the original author(s) and the source, provide a link to the Creative Commons license, and indicate if changes were made. The images or other third party material in this article are included in the article's Creative Commons license, unless indicated otherwise in a credit line to the material. If material is not included in the article's Creative Commons license and your intended use is not permitted by statutory regulation or exceeds the permitted use, you will need to obtain permission directly from the copyright holder. To view a copy of this license, visit http://creativecommons.org/licenses/by/4.0/.
\end{abstract}

(C) The Author(s) 2018

\footnotetext{
${ }^{1}$ Institute for Biophysical Chemistry, Hannover Medical School, 30625 Hannover, Germany. ${ }^{2}$ Institute of Medical and Human Genetics, CharitéUniversitätsmedizin Berlin, 13353 Berlin, Germany. ${ }^{3}$ Berlin Institute of Health, 10117 Berlin, Germany. ${ }^{4}$ Division for Structural Biochemistry, Hannover Medical School, 30625 Hannover, Germany. ${ }^{5}$ Institute for Transfusion Medicine, Hannover Medical School, 30625 Hannover, Germany. ${ }^{6}$ Department of Neuropathology, Charité-Universitätsmedizin Berlin, 10117 Berlin, Germany. ${ }^{7}$ Greenwood Genetic Center, Greenwood, SC 29646, USA. ${ }^{8}$ Institute for Clinical Chemistry and Laboratory Medicine, Medical Faculty of TU Dresden, 01307 Dresden, Germany. ${ }^{9}$ Swabian Children's Cancer Center, Children's Hospital Augsburg, 86156 Augsburg, Germany. ${ }^{10}$ Medical Genetics Center, 80335 Munich, Germany. ${ }^{11}$ Institute for Clinical Genetics, TU Dresden, 01307 Dresden, Germany. ${ }^{12}$ Core Unit for Molecular Tumor Diagnostics, National Center for Tumor Diseases Dresden, 01307 Dresden, Germany. ${ }^{13}$ Department of Pathology-Immunology, Faculty of Medicine, University of Geneva, 1211 Geneva, Switzerland. ${ }^{14}$ Department of Paediatric Haemostaseology, Medical Faculty of TU Dresden, 01307 Dresden, Germany. These authors contributed equally: Sharissa L. Latham, Nadja Ehmke. Correspondence and requests for materials should be addressed to S.L.L. (email: Latham.Sharissa@mh-hannover.de) or to D.J.M. (email: Manstein.Dietmar@mh-hannover.de) or to N.D.D. (email: Nataliya.didonato@uniklinikum-dresden.de)
} 